\title{
Cirurgia ambulatorial em hospital escola na perspectiva do paciente ${ }^{1}$
}

\author{
Guilherme Temporão Monteiro \\ Acadêmico na Universidade Positivo em Curitiba-PR \\ $\triangle$ monteiro.gt@hotmail.com \\ Catherine Potrich Cotta \\ Acadêmica na Universidade Positivo em Curitiba-PR \\ Laís Mileski Schumiguel \\ Acadêmica na Universidade Positivo em Curitiba-PR \\ Marcelo Stadler Junior \\ Acadêmico na Universidade Positivo em Curitiba-PR \\ Viviane Faccio \\ Acadêmica na Universidade Positivo em Curitiba-PR \\ Eliane Mara Cesário Pereira Maluf \\ Professora na Universidade Positivo em Curitiba-PR
}

Recebido em 3 de agosto de 2021

Aceito em 11 de novembro de 2021

\begin{abstract}
Resumo:
A cirurgia ambulatorial ainda é uma disciplina pouco presente na grade curricular de instituições de medicina no Brasil. Porém, é essencial que os acadêmicos tenham contato com essa prática na sua formação acadêmica para serem melhor capacitados para o futuro médico que lhes aguarda. Tendo em vista este cenário, o presente estudo busca, a partir da perspectiva dos pacientes, avaliar a satisfação geral destes com o serviço de Cirurgia Ambulatorial de um Hospital Escola. Foram aplicados questionários a todos os pacientes que realizaram um procedimento cirúrgico no ambulatório de Cirurgia Ambulatorial do Hospital da Cruz Vermelha nos períodos de Agosto a Novembro de $2019 \mathrm{em}$ dois momentos: imediatamente ao fim do procedimento cirúrgico e após 30 dias. Foram obtidos 183 questionários respondidos avaliando a taxa de satisfação geral do serviço, que de acordo com pacientes envolvidos no estudo, foi de $88 \%$. Além disso, em alguns aspectos, os pacientes atribuíram maiores graus de satisfação quando orientados por acadêmicos. A maioria dos pacientes estão satisfeitos com o serviço de cirurgia ambulatorial do Hospital da Cruz Vermelha do Paraná, inclusive quando esse serviço é realizado por acadêmicos. Assim, o presente estudo busca a atenção da comunidade médica para a Cirurgia Ambulatorial e incentiva outras instituições a implementarem essa matéria em suas grades curriculares.

Palavras-chave: Cirurgia ambulatorial, Satisfação do paciente, Qualidade de assistência à Saúde, Pesquisa de satisfação.
\end{abstract}

\section{Outpatient Surgery in school hospital in patient perspective}

\begin{abstract}
:
Outpatient surgery is not a very present subject in Medical Schools in Brazil. However, it is essential that the students have contact with this practice in their academic training to be better capacitated for the medical future that awaits for them. Given this scenario, this present study seeks to assess to, in patient perspective, the general satisfaction rate about Outpatient Surgery service in a School Hospital. Questionnaires were applied to all patients who underwent some outpatient surgical procedure at
\end{abstract}

\footnotetext{
${ }^{1}$ Trabalho originado do TCC intitulado "Cirurgia Ambulatorial em Hospital Escola na Perspectiva do Paciente" realizado pelos alunos de Medicina da Universidade Positivo, no ano de 2019, orientado pela Dra. Eliane Mara Cesário Pereira Maluf
} 
Hospital da Cruz Vermelha - Red Cross Hospital - between August to November of 2019 in two moments: immediately after procedure and 30 days after. 183 questionnaires were answered evaluating the general satisfaction rate, that according to the patients, was $88 \%$. Therewithal, in some aspects, patients attributed higher satisfaction rates when oriented by students. Most patients are satisfied with the quality of service of Outpatient Surgery at Hospital da Cruz Vermelha do Paraná, including when the procedures are performed by students. Therefore, this present study seeks the medical community attention to Outpatient Surgery and encourages other institutions to implement this subject on their curriculum.

Keywords: Outpatient Surgery, Patient Satisfaction, Quality of Health Care, Satisfaction research.

\title{
Cirurgía ambulatoria en un hospital universitário desde la perspectiva del paciente
}

\section{Resumen:}

La cirugía ambulatoria sigue siendo una asignatura poco presente en los planes de estudio de las instituciones médicas en Brasil. Sin embargo, es fundamental que los estudiantes tengan contacto con esta práctica en su formación académica para estar mejor formados para el futuro médico que les espera. Ante este escenario, el presente estudio pretende, desde la perspectiva de los pacientes, evaluar su satisfacción general con el servicio de Cirugía Ambulatoria de un Hospital Universitario. Se aplicaron cuestionarios a todos los pacientes que se sometieron a un procedimiento quirúrgico en la clínica de Cirugía Ambulatoria del Hospital da Cruz Vermelha en los períodos de agosto a noviembre de 2019 en dos momentos: inmediatamente al finalizar el procedimiento quirúrgico y después de 30 días. Se obtuvieron un total de 183 cuestionarios contestados, que evaluaban el índice de satisfacción global del servicio, que según los pacientes involucrados en el estudio, fue del $88 \%$. Además, en algunos aspectos, los pacientes atribuyeron un mayor grado de satisfacción cuando fueron guiados por académicos. La mayoría de los pacientes están satisfechos con el servicio de cirugía ambulatoria en el Hospital da Cruz Vermelha en el estado de Paraná, incluso cuando este servicio es realizado por académicos. Así, este estudio busca la atención de la comunidad médica hacia la Cirugía Ambulatoria y alienta a otras instituciones a implementar esta asignatura en sus planes de estudio.

Palabras clave: Cirugía ambulatoria, Satisfacción del paciente, Calidad de la asistencia sanitária, Búsqueda de satisfacción.

INTRODUÇÃO

\begin{abstract}
"A cirurgia ambulatorial é um modelo organizacional de assistência cirúrgica que permite que certos pacientes sejam tratados de forma segura e eficaz, sem internação hospitalar, recebendo alta em seu domicílio no mesmo dia da intervenção" (SANTOS et al., 2012).
\end{abstract}

$\mathrm{Na}$ década de 60, nos Estados Unidos, houve o início da era moderna da cirurgia ambulatorial. Com o apoio da anestesiologia e controles rigorosos por parte dos cirurgiões, a prática da cirurgia ambulatorial tornou-se segura, sem razões que justificassem maior incidência de intercorrências em comparação a procedimentos realizados em ambiente hospitalar. Houve um crescente aprimoramento das técnicas e práticas da cirurgia ambulatorial, também em alguns países da Europa. Entretanto, no Brasil houve pouco apoio 
formal de políticas públicas nessa perspectiva. A abordagem da cirurgia ambulatorial se deu principalmente pela vontade de agilizar o andamento das filas para procedimentos cirúrgicos. (SANTOS et al., 2012).

Tendo em vista a educação médica no Brasil, as diretrizes curriculares da graduação recomendam a inclusão da capacitação do médico generalista à realização de procedimentos clínicos e cirúrgicos. Nesse contexto, inovadoras universidades brasileiras estão adicionando a disciplina de Cirurgia Ambulatorial em suas grades curriculares visando ao aluno desenvolver técnicas operatórias apropriadas inserindo-o na realidade e dando campo para dedicação ao exercício contínuo da arte cirúrgica, aperfeiçoamento de habilidades, melhora da precisão e refinamento dos detalhes (PURIM et al., 2015).

O desempenho dos estudantes tem impacto direto sobre a impressão do paciente quanto ao procedimento realizado. 0 nível de satisfação do paciente é considerado um resultado mensurável e contribui para a avaliação da qualidade dos cuidados prestados (AYALA et al., 2011). É fundamental que aa percepções do paciente sobre a qualidade dos serviços de saúde sejam levadas em consideração, uma vez que, tem papel importante para realização de melhorias nos serviços (DIAS, 2011; MINISTÉRIO DA SAÚDE, 2013).

\section{METODOLOGIA / MATERIAL E MÉTODOS}

Por meio de um estudo observacional analítico, foram aplicados dois questionários. Os questionários passaram pelo processo de pré-teste, no qual foram aplicados a grupos de pessoas visando seu aperfeiçoamento. O primeiro questionário, em sua versão final, foi composto por 12 perguntas e foi aplicado imediatamente após o procedimento cirúrgico, por meio de um questionário impresso autoaplicável. Já o segundo questionário em sua versão final foi composto por 10 perguntas e foi aplicado 30 dias após o procedimento cirúrgico via telefone ou WhatsApp. Em ambos os questionários não havia identificação do paciente a fim de garantir o sigilo de suas respostas para que isso não afetasse o estudo. Os pacientes incluídos no estudo foram aqueles com idade maior ou igual a 18 anos, de ambos os sexos, que foram submetidos a pelo menos um procedimento cirúrgico, aceitaram participar do estudo 
e assinaram o Termo de Consentimento Livre e Esclarecido. Foram excluídos da pesquisa os pacientes que recusaram assinar o Termo de Consentimento Livre e Esclarecido, bem como pacientes que apresentavam situações que impossibilitaram a comunicação.

A amostra para o estudo contou com todos os pacientes que se submeteram a procedimentos no serviço de Cirurgia Ambulatorial do Hospital da Cruz Vermelha no período de agosto a novembro de 2019, em todos os horários de funcionamento deste ambulatório.

Dentre as variáveis abordadas em ambos os questionários, estão: gênero, idade, escolaridade, procedimento realizado e localização do procedimento. No primeiro questionário as perguntas deram enfoque à percepção do paciente quanto ao entendimento das orientações de alta dadas pelo profissional responsável e o grau de satisfação. No segundo questionário foram incluídas situações que poderiam interferir no resultado do procedimento, como tabagismo e diabetes mellitus. Foi abordada também a avaliação da satisfação quanto ao resultado estético. Em ambos os questionários, era identificado quem executou o procedimento (médico x acadêmico) e o instrumento para avaliar o grau de satisfação dos pacientes foi baseado na Escala de Likert, sendo atribuídos valores ordinais de 1 a 5, correspondendo respectivamente a insatisfeito e satisfeito. A medida de tendência utilizada para o estudo foi a mediana, visto que essa formatação de respostas permite o uso de medianas e modas (SILVA JÚNIOR, 2014). Quanto às perguntas que não correspondiam diretamente à satisfação, como por exemplo a compreensão das orientações de alta, também foram realizadas equivalências baseadas na escala de Likert.

Os dados foram testados para diferenças entre grupos a partir de testes de quiquadrado, Mann-Whitney, Krukal-Wallis (com pós teste de Dunn) e correlação de Spearman.

As análises estatísticas foram efetuadas com os pacotes estatísticos GRAPHPAD PRISM, sendo considerado um nível de significância de $5 \%(\alpha=0,05)$.

\section{RESULTADOS}

Foram convidados para responder os questionários 202 pacientes no período entre Agosto a Novembro de 2019. Dezenove deles se recusaram a responder, totalizando 183 
pacientes que responderam ao primeiro questionário, destes, 86 responderam ao segundo questionário.

Dentre o total de entrevistas realizadas em ambos os questionários, 57\% eram do sexo feminino.

Das 183 entrevistas realizadas no primeiro questionário, 92 procedimentos foram realizados por médicos, enquanto 91 foram realizados por acadêmicos. A média de idade foi 52,4 anos e o grau de satisfação geral foi de $88 \%$. Não houve relação entre a satisfação e idade, gênero, escolaridade, local do procedimento e se o procedimento foi realizado por médico ou acadêmico $(p>0,05)$.

A maior parte dos procedimentos foram na cabeça correspondendo a $36 \%$ das abordagens cirúrgicas, seguida por dorso com 18\% e membros superiores e inferiores, cada um destes representando $15 \%$.

A relação entre o grau de satisfação e o tipo de procedimento foi estatisticamente significativa $(p<0,05)$. Os pacientes submetidos a biópsia apresentaram satisfação mínima de 1 na escala de Likert, tendo a nota mínima mais baixa dentre os procedimentos, como pode ser visto na Tabela 1.

Tabela 1 - Tipo de procedimento realizado X Grau de satisfação

\begin{tabular}{lllll}
\hline Tipo de procedimento & Mediana & Mínimo & Máximo & $\mathbf{p}$ \\
\hline Biópsia & 5 & 1 & 5 & 0,036 \\
\hline Cisto sebáceo & 5 & 4 & 5 & \\
\hline Lipoma & 5 & 3 & 5 & \\
\hline Verruga/nevus & 5 & 4 & 5 & \\
\hline Outros & 5 & 3 & 5
\end{tabular}

Fonte: Própria.

Comparando o atendimento de acadêmicos e médicos, não houve diferença estatisticamente significativa quando avaliadas as orientações sobre medicações para uso em casa ou retorno ao ambulatório $(p>0,05)$, entretanto houve diferença estatisticamente 
significativa em relação às orientações sobre cuidados em casa com a ferida operatória $(p<0,05)$. Dos pacientes atendidos por médicos, 6,5\% não foram orientados e 8,7\% foram orientados parcialmente, enquanto os atendidos por acadêmicos, 4,4\% não foram orientados e apenas $1,1 \%$ orientado parcialmente, como pode ser visto na Tabela 2.

Tabela 2 - Orientações de alta X Procedimento realizado por médico ou acadêmico

\begin{tabular}{llll}
\hline Medicações & Acadêmico $(\mathbf{n}=\mathbf{9 0})$ & Médico $(\mathbf{n}=\mathbf{9 1})$ & $\mathbf{p}$ \\
\hline Não & $4,44 \%$ & $3,30 \%$ & 0,208 \\
Parcialmente & $0,00 \%$ & $3,30 \%$ & \\
Sim & $95,56 \%$ & $93,41 \%$ & \\
& & & \\
\hline Cuidados & Acadêmicos (n=91) & Médico (n=92) & $\mathbf{p}$ \\
\hline Não & $4,40 \%$ & $6,52 \%$ & 0,044 \\
Parcialmente & $1,10 \%$ & $8,70 \%$ & \\
Sim & $94,51 \%$ & $84,78 \%$ & \\
& & & $\mathbf{p}$ \\
\hline Retorno & Acadêmico (n=90) & Médico (n=92) & 0,121 \\
\hline Não & $0,00 \%$ & $4,35 \%$ & \\
Sim & $100,00 \%$ & $95,65 \%$ & \\
\hline
\end{tabular}

Fonte: Própria.

Comparando a satisfação geral dos procedimentos realizados por médicos e acadêmicos, foram encontrados valores mais baixos na escala de Likert no grupo que realizou o procedimento cirúrgico ambulatorial com médicos em relação ao grupo que realizou o procedimento com acadêmicos $(p>0,05)$.

No segundo contato, 86 pacientes responderam ao questionário. A satisfação do paciente em relação ao aspecto estético da cicatriz é independente estatisticamente do gênero, do procedimento realizado e do local da cirurgia. Também não obtendo relação estatística com tabagismo e diabetes.

A maior procura pelo serviço de cirurgia ambulatorial foi por aqueles que apresentam maior grau de escolaridade, pois cerca de $52 \%$ dos pacientes possuíam pelo menos ensino médio completo, totalizando $27,2 \%$ com ensino superior completo. Foi observada relação significativamente estatística $(\mathrm{p}<0,05)$ entre o grau de escolaridade e a satisfação estética com a cicatriz, tendo menor mediana na categoria dos entrevistados com ensino superior completo. Os pacientes com ensino fundamental completo ficaram mais satisfeitos com a 
estética da cicatriz em comparação aos pacientes portadores de ensino superior completo $(p<0,05)$. A categoria "ensino superior incompleto" foi removida das análises, pois havia apenas uma resposta nesta categoria.

\section{DISCUSSÃO / ANÁLISE DE DADOS}

Os resultados obtidos nesta pesquisa evidenciaram uma predominância de pacientes do gênero feminino, em concordância com o que foi encontrado na literatura. $O$ mesmo foi relatado em estudo realizado em um hospital regional de Londrina, que teve como objetivo caracterizar o perfil de pacientes cirúrgicos. O estudo verificou que $55 \%$ dos pacientes eram do sexo feminino (GIORDANI et al., 2014). Outro estudo realizado em um hospital universitário, entrevistou 100 pacientes cirúrgicos e também houve predominância do sexo feminino (SANTOS et al., 2012).

O perfil de pacientes que frequentaram predominantemente o ambulatório corresponde a adultos jovens, o que discorda de outro estudo que demonstrou alta predominância de idosos no uso dos serviços de saúde, fato explicado pelas condições crônicas decorrentes do envelhecimento (LIMA-COSTA, 2004).

A maior procura pelo serviço de cirurgia ambulatorial foi por aqueles que apresentam maior grau de escolaridade. Estes dados discordam do que foi concluído em estudo de LEVORATO et al., 2014 cuja procura pelos serviços de saúde foi maior por aqueles que apresentam menor grau de escolaridade e, consequentemente, rendimentos (LEVORATO et al., 2014). Outro estudo realizado no interior de São Paulo, num hospital universitário, corrobora a constatação de Levorato, no qual apenas 5,4\% da população submetida a cirurgia ambulatorial possuía ensino superior (PINTO et al., 2005). Em nosso estudo, os pacientes de menor escolaridade atribuíram notas maiores de satisfação quanto à estética da cicatriz. Tal fato sugere que, devido ao menor acesso à informação desses pacientes, possa existir uma falta de conhecimento sobre complicações associadas a ferida ou um resultado estético inesperado, apesar de não haver literatura disponível para comparação. 
A satisfação geral dos pacientes atendidos no ambulatório foi de 88\%. Tendo em vista este cenário, não há literatura similar disponível para fins de comparação. Essa informação, no entanto, sustenta os propósitos deste estudo e ainda impulsiona outras instituições de ensino médico à expandir seus horizontes quanto à implementação da disciplina de Cirurgia Ambulatorial nas suas grades curriculares.

Os pacientes que receberam orientações por acadêmicos atribuíram graus de satisfação maiores do que aqueles que receberam as mesmas orientações pelos médicos. Não havendo literatura disponível para fins de comparação, foi levantada a hipótese de que, pelo fato de o acadêmico estar em um ambiente de aprendizado, ele dispõe de mais tempo para realizar o procedimento e orientar sobre a alta, além de ser acompanhado diretamente por um preceptor, o que possivelmente melhora a relação médico-paciente, fazendo com que o paciente atribua notas maiores nesse critério.

Os pacientes submetidos à biópsia apresentaram menor nota mínima de satisfação na escala de Likert, em comparação aos outros procedimentos realizados. Isso pode estar relacionado com a relação médico-paciente, uma vez que a biópsia traz mais estresse ao paciente por haver suspeita de malignidade. As peculiaridades do diagnóstico de câncer impõem ao médico abordagem diferenciada, tornando necessário a abordagem dos aspectos psicossociais do doente de modo a não gerar inseguranças (BASTOS et al, 2017).

A principal limitação encontrada no estudo foi a dificuldade de comunicação com os pacientes na segunda etapa da coleta de dados. Essa queda, no entanto, não invalida o estudo, visto que está de acordo com a literatura. Em material publicado por Mattar, observa-se que a quantidade de dados e o índice de repostas obtidos em uma entrevista pessoal eram altos, enquanto a quantidade de dados obtidos em pesquisa via telefone era baixa (MATTAR, 1999).

\section{CONCLUSÃO / CONSIDERAÇõES FINAIS}

A maioria dos pacientes estão satisfeitos com o serviço de cirurgia ambulatorial do Hospital da Cruz Vermelha do Paraná. Além disso, pacientes sentem-se melhor orientados por acadêmicos. Tais fatos corroboram o bom aproveitamento dos alunos e bons resultados 
apresentados na disciplina curricular de cirurgia ambulatorial. Tal fato pode servir como incentivo para que outras instituições de ensino implementem tal disciplina em suas grades curriculares.

O perfil de pacientes que frequentaram o ambulatório durante o estudo é de pacientes do sexo feminino na sua quinta década de vida com ensino igual ou superior ao ensino médio completo.

O grau de satisfação quanto à estética da cicatriz é influenciada diretamente pelo grau de escolaridade.

Logo, é de suma importância que mais pesquisas sobre o tema sejam realizadas de modo que a Cirurgia Ambulatorial caminhe para se tornar matéria essencial na formação do acadêmico de medicina.

\section{REFERÊNCIAS}

AYALA, S.; CRISTIANI, F.; SARALEGUI, J. Satisfação do paciente em um serviço de anestesia ambulatorial. Anest Analg Reanim , Montevidéu, v. 24, n. 2, p. 53-58, 2011. Disponível em <http://www.scielo.edu.uy/scielo.php?script=sci_arttext\&pid=S1688-12732011000200003\&lng=en\&nrm=iso>.

BASTOS, L. O.; ANDRADE, E. N.; ANDRADE, E. O. Relação médico-paciente na oncologia: estudo a partir da perspectiva do paciente. Revista Bioética, 2017.

DIAS, O. V.; VIEIRA, M. A.; DIAS, J. P.; RAMOS, L. H. As dimensões da satisfação dos usuários do Programa Saúde da Família: confiabilidade e empatia. Acta paul. enferm., São Paulo, v. 24, n. 2, p. 225-231, 2011. Available from <http://www.scielo.br/scielo.php?script=sci_arttext\&pid=S0103-21002011000200011\&lng=en\&nrm=iso>.

GIORDANI, A. T.; SONOBE, H. M.; EZAIAS, G. M.; VALÉRIO, M. A.; BARRA, M. R.; STADLER, D. B. Profile of surgical patients treated in a public hospital. Journal of Nursing UFPE on line, [S.1.], v. 9, n. 1, p. 54-61, oct. 2014. ISSN 1981-8963. Disponível em: <https://periodicos.ufpe.br/revistas/revistaenfermagem/article/view/10306>.

LEVORATO, C. D.; MELLO, L.M.; SILVA, A. S.; NUNES, A. A. et al . Fatores associados à procura por serviços de saúde numa perspectiva relacional de gênero. Ciênc. saúde coletiva, Rio de Janeiro , v. 19, n. 4, p. 12631274, Apr. 2014 . Available from <http://www.scielo.br/scielo.php?script=sci_arttext\&pid=S1413$\underline{81232014000401263 \& \operatorname{lng}=e n \& n r m=\text { iso>. }}$

LIMA-COSTA, M. F. A influência da idade e da escolaridade no uso de serviços preventivos de saúde - Inquérito de Saúde da Região Metropolitana de Belo Horizonte, Minas Gerais, Brasil. Epidemiologia e Serviços de Saúde 2004.p.209-215.

MATTAR, F. N. Pesquisa de Marketing: metodologia e planejamento. São Paulo: Editora Atlas, 1999. 337 p., Volume 1, 5a edição.

MINISTÉRIO DA SAÚDE (BR). Secretaria-Executiva. Núcleo Técnico da Política Nacional de Humanização. Política Nacional de Humanização. Brasília: Ministério da Saúde; 2013. 
PINTO, T. V.; ARAUJO, I. E.; GALLANI, M. C. Enfermagem em cirurgia ambulatorial de um hospital escola: clientela, procedimentos e necessidades biológicas e psicossociais. Rev. Latino-Am. Enfermagem, Ribeirão Preto , v. 13, n. 2, p. 208-215, Apr. 2005 Disponível em: <http://www.scielo.br/scielo.php?script=sci_arttext\&pid=S0104-11692005000200012\&lng=en\&nrm=iso>.

PURIM, K. S.; JAMES, S.; FERNANDES, J. W. Basic skills for outpatient surgery in medical graduation. Revista do Colégio Brasileiro de Cirurgiões, [s.l.], v. 42, n. 5, p.341-344, out. 2015. FapUNIFESP (SciELO).

SANTOS, M. A.; ROSSI, L. A.; PAIVA, L.; DANTAS, R. A.; POMPEO, D. A.; MACHADO, E. C. Medida da ansiedade e depressão em pacientes no pré-operatório de cirurgias eletivas. Revista Eletrônica de Enfermagem, Ribeirão Preto, v. 7, n. 922, p.222-227, out. 2012. Disponível em: https://www.fen.ufg.br/fen_revista/v14/n4/pdf/v14n4a21.pdf.

SILVA JÚNIOR, S. D. Mensuração e Escalas de Verificação: uma Análise Comparativa das Escalas de Likert e Phrase Completion. Revista Brasileira de Pesquisa de Marketing Opinião e Mídia, Bayeux, v. 15, n. 1, p. 1-16, 1 mar. 2014.

\section{CONTRIBUIÇÕES DOS AUTORES}

Todos os autores participaram ativamente nos seguintes processos: Concepção inicial do projeto; Revisão bibliográfica; Elaboração de material (questionários); Coleta de dados; Análise estatística de dados; Escrita do manuscrito; Revisão do manuscrito; Elaboração do manuscrito final.

\section{(c)) Br}

Este trabalho está licenciado com uma Licença Creative Commons - Atribuição 4.0 Internacional. 